\title{
Las Maretas de Lanzarote: arqueología industrial del agua
}

\author{
The ponds of Lanzarote: industrial archeology of water \\ Rodríguez-de Vera BC ${ }^{(1)}$, Hernández-Martínez $\mathrm{FJ}^{(2)}$, Jiménez-Díaz $\mathrm{JF}^{(1)}$, \\ Jiménez-Rodríguez $\mathrm{I}^{(3)}$ \\ (1)Universidad de Las Palmas de Gran Canaria, Canarias, España \\ ${ }^{(2)}$ Cabildo Insular de Lanzarote, Lanzarote, España \\ ${ }^{(3)}$ The Ostelea School of Tourism \& Hospitality - Universitat de Lleida, España \\ bienvenida.rodriguez@ulpgc.es
}

\section{Resumen}

El agua ha sido, y sigue siendo, un factor clave para el desarrollo socioeconómico de la isla de Lanzarote. En la época prehispánica y durante los primeros siglos de la conquista, el principal aprovechamiento eran las aguas subterráneas, siendo también muy importante el aprovechamiento de las de lluvia que se recogían de diversas maneras, por ejemplo, mediante alcogidas, maretas y aljibes; procedimientos que se utilizaron hasta 1964 cuando se instaló la primera planta desaladora en la isla.

La mareta es un tipo de depósito, se trata de una hondonada natural o excavada cerca de una montaña para recoger el agua que discurría por las laderas. Las maretas suelen poseer un muro exterior de piedra enfoscada de cal. La mayor parte de las maretas se concentraba en la zona que fue cubierta de material volcánico tras las erupciones de Timanfaya en el siglo XVIII; siendo la más importante para la isla la "Gran Mareta" de Teguise que fue construida sobre una charca antigua en el S. XV y se utilizó hasta 1963. Su diámetro era de $40 \mathrm{~m}$. con una profundidad de $9 \mathrm{~m}$. y capacidad de $40.000 \mathrm{~m} 3$. En el año 1902, tras varios años de sequía, se había iniciado la construcción de las denominadas "Maretas del Estado", una construcción cuya finalidad era asegurar el suministro a la capital de la isla. Hubo muchos problemas con las obras y éstas quedaron sin terminar por falta de fondos, hasta que en su visita a la isla, el Rey Alfonso XIII, en 1906 concedió una ayuda para su finalización en 1912. Las maretas ocupan una superficie de $90.000 \mathrm{~m} 3$ (con $3900 \mathrm{~m} 3$ para los 16 aljibes y el resto como zona de alcogida). Cada aljibe mide $31 \mathrm{~m}$ de largo, $6 \mathrm{~m}$ de ancho y $6 \mathrm{~m}$ de alto. En la actualidad esa superficie ya no queda tan grande, porque sobre ella se ha trazado la vía de circunvalación de Arrecife. En 1997 se inició el Proyecto Maretas por parte del Cabildo Insular de Lanzarote para restaurar dichas obras de arqueología industrial del agua y convertir la zona de recogida de aguas en un gran espacio de cultura contemporánea. 
Palabras clave: Maretas. Lanzarote, arqueología industrial. agua

Key words: Ponds, Lanzarote, archeology industrial, water

\section{Referencias}

Gil González, Jaime (2005): Los Cultivos Tradicionales de la isla de Lanzarote. Los Granos: Diversidad y Ecología. Cabildo de Lanzarote y La Caja de Canarias. La Laguna. $253 \mathrm{pp}$.

González Morales, A. (2003): "La cultura del agua en Lanzarote". Ponencia en las Jornadas del Agua de la S.C.R.D. TORRELAVEGA, Arrecife.

Hernández Gutiérrez et al. (1999): Patrimonio Histórico de Arrecife de Lanzarote. Cabildo de Lanzarote. Arrecife.

Montelongo Fránquiz, Antonio y FALERO LEMES, Marcial Alexis (2000): "El agua en Arrecife, condicionante histórico". En IX Jornadas de Estudios sobre Fuerteventura y Lanzarote, 20-24 de septiembre de 1999. Puerto del Rosario. Tomo I. Servicio de Publicaciones de los Cabildos de Fuerteventura y Lanzarote, pp. 162/172.

Peña Hernández, Marta (2005): "Breve aproximación a los sistemas agrícolas de Lanzarote”. En Rincones del Atlántico. N², pp. 220/227. 